\title{
THE PARTOGRAPH: AN ESSENTIAL YET UNDERUTILIZED TOOL
}

Beenu Kushwah ${ }^{1}$, Alok Pratap Singh², Shipra Singh ${ }^{3}$.

1. Assistant Professor, Department of Obstetrics \& Gynaecology Shyam Shah Medical College

2. Assistant Professor, Department of Anesthesiology, Shyam Shah Medical College

3. Post Graduate Student, Department of Obstetrics \& Gynaecology Shyam Shah Medical College

\section{CORRESPONDING AUTHOR:}

Dr Beenu Kushwah,

F-5, Doctor's Colony,

SGMH Campus, Huzur, Rewa

Pin-486001

E-mail: drbeenukushwah@gmail.com

\section{HOW TO CITE THIS ARTICLE:}

Beenu Kushwah, Alok Pratap Singh, Shipra Singh. "The Partograph: an Essential Yet underutilized Tool”. Journal of Evolution of Medical and Dental Sciences 2013; Vol2, Issue 24, June 17; Page: 4373-4379.

INTRODUCTION: Maternal Mortality and Infant Mortality Reduction has been a goal of several International and National Welfare programmes for more than 2 decades now. Most of the maternal and neonatal morbidities are avoidable by applying very basic principles to our day to day practice and by revisiting the basic practices. Revitalization of partograph is one of them, which in spite of being very helpful to avoid the maternal and neonatal complications related with prolonged and obstructed labour; not being utilized properly. OBSERVATIONS: WHO has long back labeled Partograph as an essential tool to prevent maternal and neonatal complications, still its use does not seem to be worldwide. By reviewing the literature it has been observed that main hindrance in the use of this useful tool is related with the management and administration issues including logistic problems, shortage of staff, training and supervision inadequacy and inadequate attention being given to its inclusion as an essential labour monitoring tool starting from the learning periods of Nurses and Doctors. CONCLUSION: Partograph use needs to be encouraged more in countries like India where we wish to utilize peripheral staff and non obstetricians as a workforce for management of pregnant females. As we do not have effective peripheral level emergency obstetrics services, it is very crucial that every labouring woman should be referred at proper time in order to improve maternal and neonatal outcomes which can only be achieved by proper monitoring of labour by using a simple tool like Partograph.

According to Registrar General Of India, the Millennium Development Goals (MDG) of the United Nations has set the target of achieving 200 maternal deaths per lakh of live births by 2007 and 109 per lakh of live births by 2015 (1). For 2008, there were an estimated 358, 000 maternal deaths in the world, or a maternal mortality ratio (MMR) of 260 maternal deaths per 100,000 live births (2). Of the estimated total of 358,000 maternal deaths worldwide, developing countries accounted for $99 \%(355,000)$. Nearly three fifths of the maternal deaths $(204,000)$ occurred in the sub-Saharan Africa region alone, followed by South Asia (109,000). Thus, sub-Saharan Africa and South Asia accounted for $87 \%$ (313,000) of global maternal deaths. By country, India had the largest number of maternal deaths. India has shown an impressive progress in reducing its maternal

Journal of Evolution of Medical and Dental Sciences/ Volume 2/ Issue 24/ June 17, 2013 Page 4373 


\section{REVIEW ARTICLE}

mortality ratio; it is worth noting that the number of States that have realized MDG target in 20072009 has gone up to 3 against 1, Kerala which was the sole State with this distinction in 2004-2006. Tamil Nadu and Maharashtra are the new entrants. Andhra Pradesh, West Bengal, Gujarat and Haryana are in closer proximity to the MDG target. However, Empowered action group (EAG) states and Assam are still lagging far behind with a subtotal MMR of 308 against the National MMR of 212 (1).With only Three years left until the 2015 deadline to achieve the MDGs, closer examination of maternal mortality levels and the interventions required to reduce it needs to be done. Repeated assessments of current practices towards reaching the set goals are needed for planning of reproductive health programmes and to guide advocacy efforts and research at the national level.

Amongst the most common causes of Maternal Mortality, prolonged labour and obstructed labour remain major causes; they can lead to ruptured uterus, postpartum haemorrhage, infection, obstetric fistula, and foetal injury or death. Information about prolonged and obstructed labour is often underreported. The incidence of these conditions varies widely, ranging from as low as $1 \%$ in some populations to as high as $20 \%$ in others; in 2000 , about 42,000 deaths, or $8 \%$ of maternal deaths, were attributed to prolonged and obstructed labour (3). As registration system is often poor in settings where prolonged labour, obstructed labour, and maternal deaths are common. In total, obstructed labour and puerperal sepsis can contribute to up to $25 \%$ of maternal deaths in India which is directly related to poor attention given to watch the progress of labour and improper management of prolonged labour (4).

Partograph is a Greek word meaning "Labour Curve" (5). Partograph comes as a pre-printed one-page form on which labour observations are recorded, it provides a graphic overview of the progress of labour and records information about maternal and foetal condition during labour. Partograph is considered to be a very effective tool to monitor labour and prevent prolonged and obstructed labour. It provides information about deviations from the normal progress of labour and about abnormalities of maternal or foetal condition during labour. It provides timely information regarding further intervention in the form of referral to a higher-level facility, labour augmentation, and caesarean section etc. depending upon the resources available. At the same time it facilitates ongoing evaluation of the effects of these interventions (5). World Health Organization has designated management of labour with the Partograph as one of the essential elements of obstetric care at the first referral level (6). Although the basic idea of devising partograph was to prevent complications of prolonged labour at peripheral health care centres by providing them an easy tool to monitor the progress of labour which could even be used by the midwives and non obstetrician doctors, mostly its use has been reduced to only as a teaching tool to be used in tertiary level health care centres and Medical Colleges thereby defeating its very purpose of invention.

ORIGIN AND EVOLUTION OF PARTOGRAPH: There are many versions of the Partograph available today. Friedman's labour curve is a crude version that is used by many midwives and healthcare professionals (7). Philpott's Partograph is an improved version of Friedman's labour curve and was developed to meet maternal health needs in Harare, Zimbabwe $(8,9,10)$. The WHO developed its own Partograph as an adaptation of the Philpott Partograph (11), it includes a latent phase of 8 hours and active phase starts at $3 \mathrm{~cm}$ cervical dilation (Picture 1). A simpler and more user friendly second version of it was created in 2000 (Picture 2), removing the latent phase, active phase starting at $4 \mathrm{~cm}$ cervical dilation (12). The latest version is the simplified one, designed especially for use by skilled

Journal of Evolution of Medical and Dental Sciences/ Volume 2/ Issue 24/ June 17, 2013 Page 4374 


\section{REVIEW ARTICLE}

attendants in health centres, it excludes the latent phase and descent of the presenting part; the active phase starts at $4 \mathrm{~cm}$ cervical dilation, for making it further simpler it is made colour coded (WHO, 2006; Mathai, 2009). In 1991 in Asia, the WHO examined over 35,000 births in the largest trial of the Partograph ever done (13). The study found evidence that prolonged labour, postpartum sepsis, and stillbirth were significantly reduced when the Partograph was used. Augmentation rates and caesarean rates were also reduced. In the 1970s in Zimbabwe, Philpott and Castle also reported positive outcomes. Although only a small number of controlled and quasi-controlled studies has examined the impact of Partograph use on labour outcomes. However, other uncontrolled and generally smaller studies have reported that the tool has had a positive impact on maternal and perinatal health outcomes, especially in low-resource settings (14). Although the partograph has been viewed as an important tool in obstetric care. Current levels of knowledge and use are low in developing countries $(13,14)$. This means that the progress of labour may not be monitored at all or that labour monitoring may not translate into the emergency actions required when complications arise. To be effective, the Partograph requires a strong supervisory component $(15,16)$. The Partograph may have quality-of-care benefits that go beyond effective labour monitoring and management, including improved continuity of care and increased interaction between health care providers and the labouring woman $(17,18,19)$. In developing countries, most poor women and many other women give birth at home without the assistance of a skilled birth attendant or any formal monitoring of labour progress (20). As we are encouraging skilled birth attendants to be available at every birth taking place; even at home, a special colour coded Partograph has been developed by WHO which can easily be used by non medical professionals (12).

\section{PROBLEMS FACED IN USE OF THE PARTOGRAPH:}

1. Understaffed Health care centres: Shortage of human resources is a chronic challenge for challenge for health services in low-resource settings. Rarely are there enough personnel with the needed skills and knowledge, and labour wards are often dangerously understaffed. Thus, Health Manager and Policy makers should make efforts to ensure availability of health care staff to implement the proper use of Partograph.

2. Inefficient Referral Linkage: In most low-resource settings, the lack of functioning referral mechanisms presents a major challenge to effective use of the Partograph. If emergency transportation from community to hospital is nonexistent or functions poorly, the health provider is often unable to act on Partograph findings and to transfer a woman for timely intervention when complications arise. In addition, labouring women referred from health centres as emergency cases often arrive at tertiary facilities without any documentation to provide crucial information about the preceding hours or days of their labour. Even when providers have started Partograph monitoring at home or in a health centre, these Partographs are often discarded when the woman arrives at an emergency centre, and a new one is started from the time of her arrival.

3. Logistics problem: To begin with, institutions must have the basic financial resources to ensure a dependable supply of Partograph forms as this has been quoted as one of the important factor responsible for its non-use in day to day practice.

4. Poor knowledge and lack of ongoing in-service trainings: Use of Partograph requires underlying clinical competence in labour management and resulting complications. It has been observed that Nursing and midwifery curriculum does not provide sufficient knowledge to use it

Journal of Evolution of Medical and Dental Sciences/ Volume 2/ Issue 24/ June 17, 2013 Page 4375 


\section{REVIEW ARTICLE}

effectively in peripheral settings, neither is its significance is properly conveyed to the students. Most pre-service training sites are based in urban teaching hospitals and institutes of education, far from the settings in which most students are likely to eventually practice. Nursing, midwifery, and medical students often have few opportunities to hone their Partograph skills in real-world environments. Furthermore, if providers have not acquired competency in Partograph use during pre-service training, it is unlikely that they will become proficient as a result of short, one-off in-service training programs. Even if the staff is trained for using the Partograph, they are not supported with inservice trainings and assessment of their practices to get feedbacks.

5. Too much records to keep: In many health systems where paper records are available, the customary way of capturing patient information is the written narrative. Such documentation tends to be subjective in nature and to lack standardization; it is less detailed, specific, and objective than the Partograph. For health care providers accustomed to conventional medical records, the graphic format of the Partograph and the plotting skills it requires may be difficult both to understand and to use. While the tool appears simple, providers may lack the underlying skills and knowledge that it requires. Also, health care providers may be reluctant to record medical events in detail, for fear that their performance will later be found wanting.

There is a good example of relieving the record keeping burden; one facility in Bangladesh faced challenges with record keeping in general and with the Partograph in particular. When the staff analyzed the situation, they found that providers were required to complete 11pieces of paper per patient; thus, the facility decided to consolidate some records. As a consequence of this streamlining, completion and use of the Partograph improved.

\section{ADDRESSING THE PROBLEMS}

Training: Although it's been almost 20 Years since WHO recognized Partograph as an essential tool in labour monitoring and management, its use has been very inconsistent and incorrect. Providers often lack the underlying knowledge and skills required to manage labour and delivery; Training of using Partograph in a right way should be in place right from the under graduation courses of Midwives and Doctors while they are learning the basics of Labour room protocols. Students should be made maintaining the Partograph records themselves under supervision and be told about the necessary intervention if required. After joining the medical services as obstetrician or Midwives at peripheral centres they should be encouraged to refresh their knowledge regarding Partograph maintenance according to facilities and environment of their work places through undertaking inservice trainings. Training should address competency within the facility, not just among individual providers. For the Partograph to be used correctly, all key members of the maternity care team must be trained and clinically competent to assess cervical dilation, to accurately plot dilation on the Partograph, and to analyze and use the data to make decisions about referral and action.

(To help address Partograph training issues, the World Health Organization has published WHO Partograph: E-Learning Tool (2010). This CD-ROM is designed to be used for self-learning or as an aid in classroom learning in pre-service and in-service midwifery and medical training)

Adequate supply of recourses: Apart from the health care providers, administrators should also understand the importance of providing basic infrastructure and resources that are needed to make it a routine practice. Frequent non-availability of resources creates frustration amongst health care workers and causes an undesired break of practice, further causing inconsistent use of this

Journal of Evolution of Medical and Dental Sciences/ Volume 2/ Issue 24/ June 17, 2013 Page 4376 
important tool. It should be considered as a part of routine medical record maintenance practices. Under supply of a mere pre-printed form, which doesn't cost much but could be a life saving tool for both the mother and baby, should never be a hindrance in the use of partograph.

Provision of Patronship: By now we have understood the importance of ongoing training and support to the staff in case of any difficulty arises. It is desirable that the trainee have access to a clinically competent patron who can reinforce learning, assess performance, and promote improvement without casting blame. In the absence of patron, providers find it difficult to use Partographs consistently and correctly. These patrons can be chosen from the nearest teaching institutes. Patrons are essential; professionals who take this role must themselves be properly trained and have dedicated time to devote to it. Patron ship should be completely volunteered and not to be added on the already heavy duties of individuals who are not willing to take this role.

CONCLUSION: The Millennium Development Goals (United Nations, 2011) have focused renewed attention on infant and maternal mortality. Revitalization of the Partograph can play an important role in helping to achieve those goals. Many organizations will need to collaborate to conduct the necessary Partograph research and to advance labour monitoring techniques and emergency obstetric care. Professional associations; bilateral and multilateral institutions; governmental and non-governmental organizations; medical, nursing, and midwifery schools; and health facilities all have a role to play in improving labour and delivery practices. Promoting use of Partograph as a routine in labour management will be a major step towards reducing Maternal and Neonatal Morbidity and Mortality.

To address the challenges for using Partograph amongst health care providers, health systems must establish an environment that supports its correct and consistent use. To realize the real potential of this wonderful tool we first need to address the root level problems starting with the revision of current mid-wifery and medical under graduation course, giving more importance to its implementation part and making students recording Partograph themselves in Labour wards, other solutions would be availability of adequate resources and support that staff needs by asking them about their difficulties at their work places, last but not the least keeping the health care staff updated by providing in service trainings and appraisal of their practices at their workplaces itself.

\section{REFERENCES:}

1. Sample Registration System (SRS) Office of Registrar General, India, 7th July 2011.

2. Trends in Maternal Mortality: 1990-2008.Estimates developed by WHO,UNICEF,UNFPA\& World Bank WHO Library 2010.

3. Mathai, M. 2009. The partograph for the prevention of obstructed labour. Clinical Obstetrics and Gynecology 52(2):256-269.

4. World Health Organization. The World Health Report 2005: Make every Mother and Child count. Geneva: World Health Organization; 2008.

5. Allauddin Md, Runal Bal, Arunangsu De, Parthajit Mandal, Mayoukh Chakraborty. Monitoring labour with WHO Modified Partograph- A study report. NJOG 2008; 3: 8-11.

6. Friedman, E.A. 1954. Graphic analysis of labour. American Journal of Obstetrics and Gynecology 68(6):1568-1575.

Journal of Evolution of Medical and Dental Sciences/ Volume 2/ Issue 24/ June 17, 2013 Page 4377 
7. Philpott RH. Graphic Records in Labour. BMJ 1972; 4: 163-165.

8. Philpott RH, Castle WM. Cervicographs in the management of labour in primigravidae. I. The alert line for detecting abnormal labour. J Obstet Gynecol Br Cwlth 1972; 79: 592-598.

9. Philpott RH, Castle WM. Cervicographs in the management of labour in primigravidae . II. The action line and treatment of abnormal labour. J Obstet Gynecol Br Cwlth. 1972; 79: 599600 .

10. World Health Organization. World Health Organization partograph in the management of Labour. Lancet 1994; 343: 1399-1404.

11. World Health Organization. 1991. Essential elements of obstetric care at the first referral level. Geneva.

12. Mathews J.E., Rajaratnam A, George A, Mathai M, Comparison of the WHO Partographs. International Journal Gynecology and Obstetrics 2007; 96: 147-50.

13. World Health Organization. 1994a. The application of the WHO partograph in the management of labour. Report of a WHO multicentre study 1990-1991. Geneva: WHO Maternal Health and Safe Motherhood Programme.

14. Lavender, T., Hart, A., and Smyth, R.M.D. 2008. Effect of partogram use on outcomes forwomen in spontaneous labour at term. Cochrane Database of Systematic Reviews Issue 4, Art. No. CD005461.

15. Pettersson, K.O., Svensson, M.L., and Christensson, K. 2000. Evaluation of an adapted model of the World Health Organization partograph used by Angolan midwives in a peripheral delivery unit. Midwifery 16(2):82-88.

16. Fahdhy M, Chongsuvitwong M. Evaluation of World Health Organisation partograph. Midwifery implementation by midwives for maternity home birth in Medan, Indonesia. Midwifery, 2005; 21: 301-10.

17. World Health Organization. 1994b. Preventing prolonged labour: A practical guide. The partograph. Part I: Principles and strategy. Geneva.

18. American College of Nurse-Midwives. 2008. Life saving skills: Manual for midwives, $4^{\text {th }}$ ed. Silver Spring, MD. Bergstrom, S. 2001

19. Lavender, T., Lugina, H., and Smith, H. 2007. The partograph: A life-saving tool for African midwives. Tropical Doctor 37(3):191-192.

20. Montagu, D., et al. 2011. Where do poor women in developing countries give birth? A multicountry analysis of Demographic and Health Survey data. PLoS ONE 6(2):e17155. 


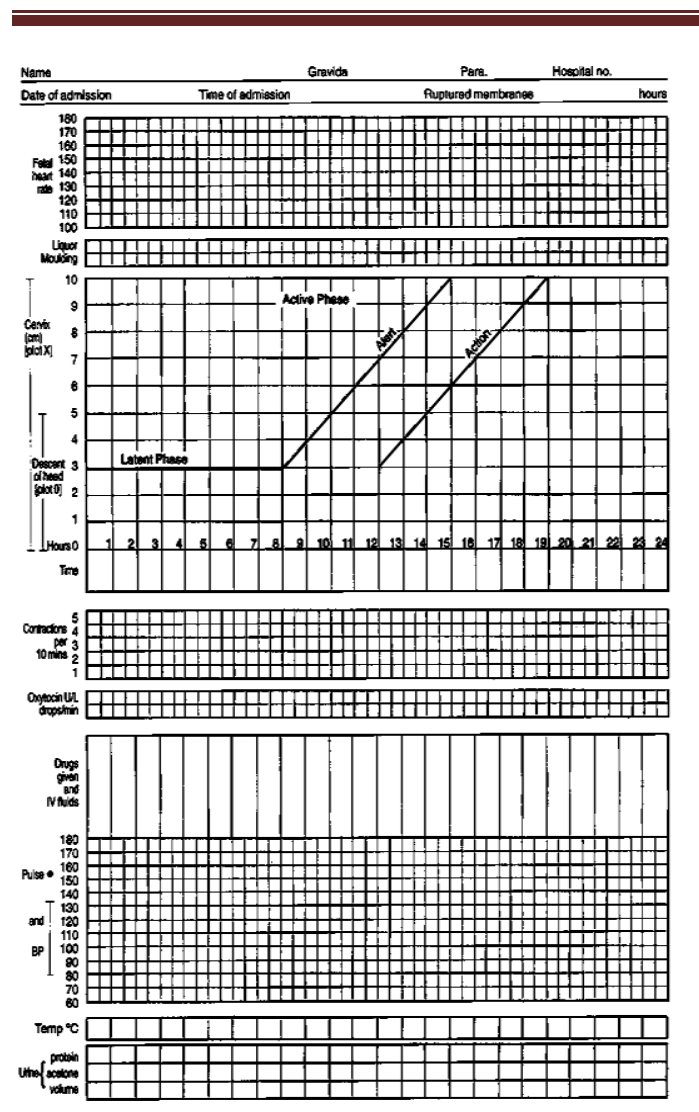

Picture-1

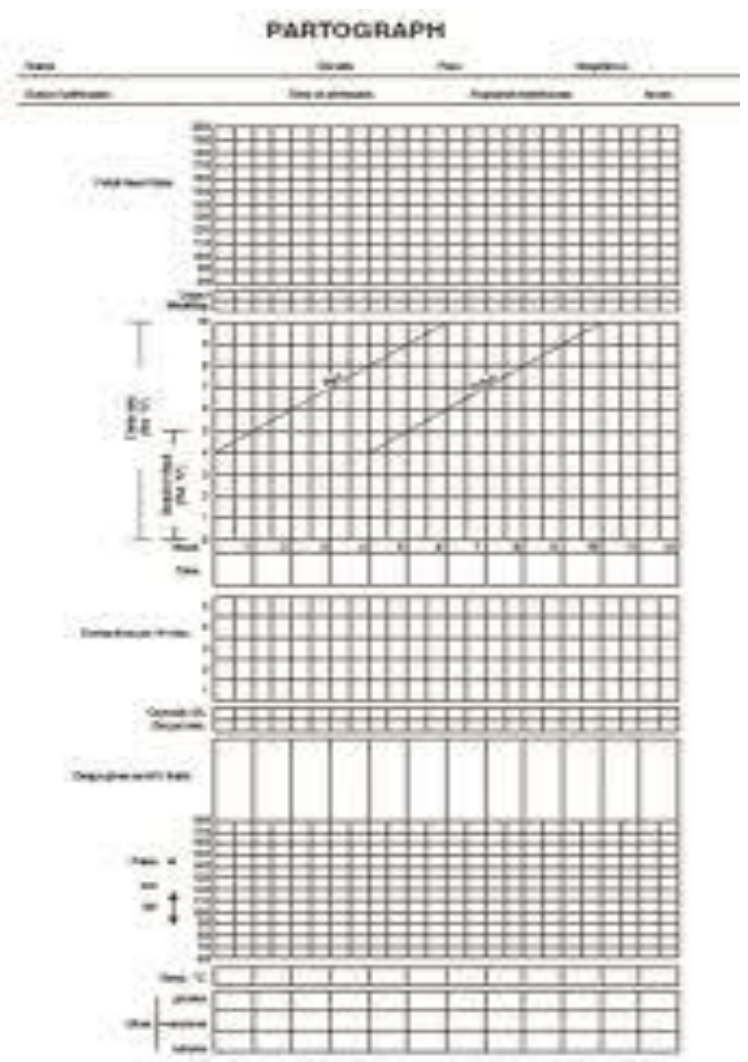

Picture-2

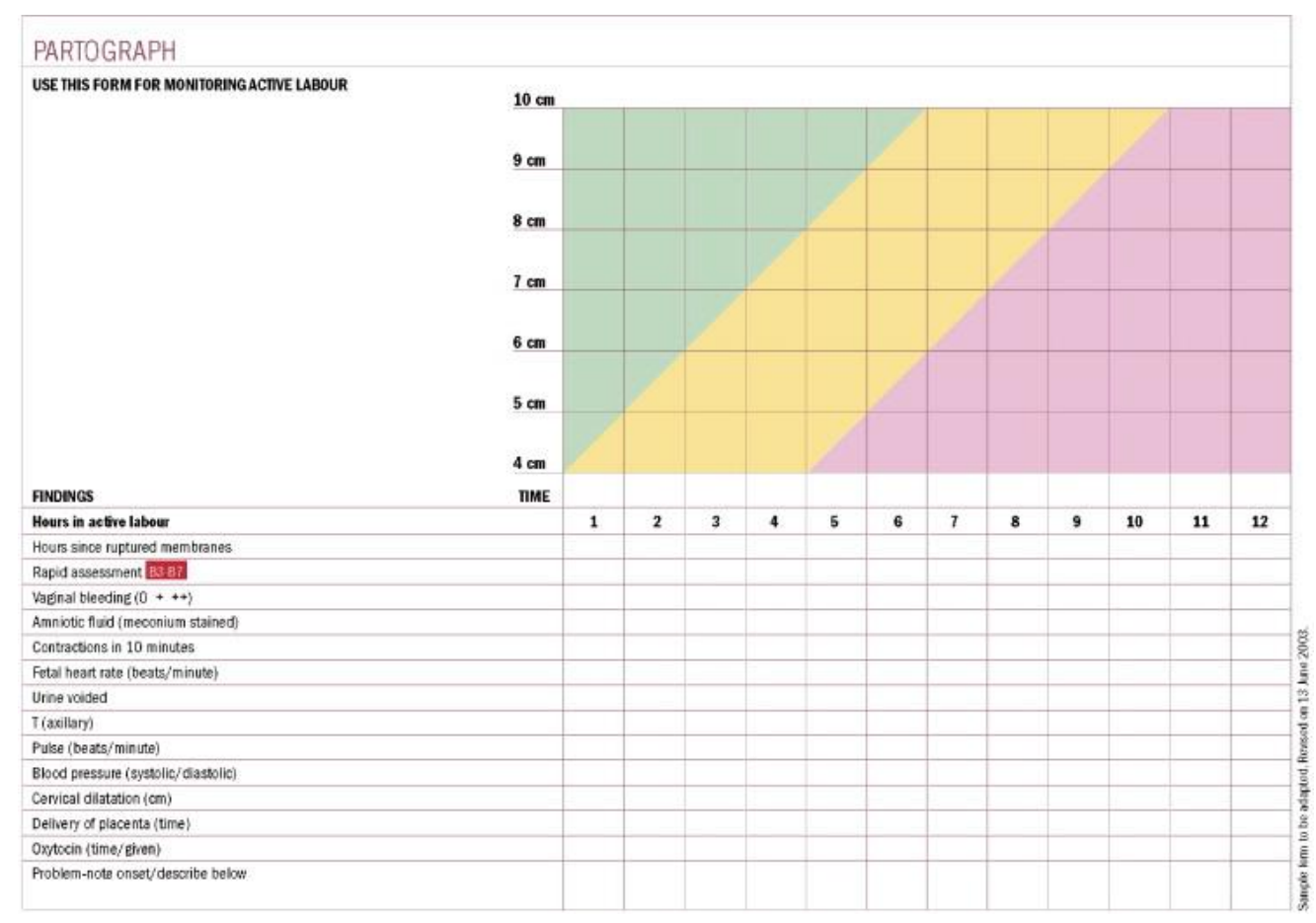

Picutre-3

Journal of Evolution of Medical and Dental Sciences/ Volume 2/ Issue 24/ June 17, 2013 Page 4379 